Research Article

\title{
Constitutions of Deficiency and Stasis of Traditional Chinese Medicine and Related Factors among Middle-Aged Women in Taiwan
}

\author{
Ping-Ho Chen $\mathbb{D}^{\mathbb{D}},{ }^{1}$ Sheng-Miauh Huang $\mathbb{D}^{2},{ }^{2}$ Jerry Cheng-Yen Lai $\mathbb{D},{ }^{3}$ and Pei-Jung Yu $\mathbb{C}^{2}$ \\ ${ }^{1}$ Department of Traditional Chinese Medicine, Taipei Medical University Hospital, Taipei, Taiwan \\ ${ }^{2}$ Department of Nursing, Mackay Medical College, New Taipei City, Taiwan \\ ${ }^{3}$ Department of Medical Research, Taitung MacKay Memorial Hospital, Taitung, Taiwan
}

Correspondence should be addressed to Sheng-Miauh Huang; r910862@yahoo.com.tw

Received 24 March 2020; Revised 26 August 2020; Accepted 29 September 2020; Published 20 October 2020

Academic Editor: Arthur De Sá Ferreira

Copyright $\odot 2020$ Ping-Ho Chen et al. This is an open access article distributed under the Creative Commons Attribution License, which permits unrestricted use, distribution, and reproduction in any medium, provided the original work is properly cited.

Background. Traditional Chinese medicine (TCM) appears to be the common therapy in middle-aged women. The constitution serves as a guide for TCM treatment. However, little is known about the constitution and related factors in middle-aged women. The objectives of this study were to describe the yang-deficiency, yin-deficiency, and stasis constitutions in middle-aged women. Demographic and health factors related to yang deficiency, yin deficiency, and stasis were also examined. Methods. A total of 1,000 women aged 40-65 years were selected from 2009 through 2018 using random sampling from the Taiwan Biobank Research Database in Taiwan. Yang-deficiency, yin-deficiency, and stasis were assessed using the body constitution questionnaire. Multiple logistic regression analysis was used to identify factors associated with constitution in deficiency or stasis. Results. The proportions of middle-aged women who had the constitution in yang-deficiency, yin-deficiency, and stasis were $29.7 \%, 21.7 \%$, and $17.7 \%$, respectively. The result of binary logistic regression showed that current menstruation, abnormal spirometry, and education level were predictive factors of yang deficiency. Women with younger age, abnormal spirometry, or a vegetarian diet had a significantly associated yin deficiency. Younger age, abnormal spirometry, and coffee habit were predictors of stasis. Conclusions. Middle-aged women in Taiwan with abnormal spirometry had a higher risk for deficiency or stasis constitutions, especially for those younger than 56 years. Healthcare providers should learn patients' constitutions and provide appropriate advice, referring them to safe providers of their desired method.

\section{Introduction}

The identification of constitution, a concept that has been developed in the 1970s in China, provides basic guidance in the traditional Chinese medicine (TCM) diagnostic and therapeutic systems. A constitution can be viewed as the individual's body condition that makes an individual susceptible to certain diseases but not others. The literatures indicate patients with different constitutions are treated differently [1-3]. The basis of the yin-yang theory in TCM is that yin and yang are the two opposite, complementary, and interrelated cosmic forces found in all matter in nature. All changes that are observed in the world, including those of the human body, arise from the ceaseless motion of both yin and yang. If yin-yang has deviated or is out of balance, some psychophysiological symptoms and patterns appear, such as deficiency and stasis $[4,5]$. The deficiency in constitutions include yang deficiency and yin deficiency. The former is marked by a low energy (qi) level in the physiological functioning of the body. The latter implies that a person has insufficient yin fluid [4-6]. A stasis indicates that the dynamic yin and yang interactions are slowed or deficient, which usually happens in patients with a disease because their qi and blood are obstructed by the lesion site, which is prevalent among postmenopausal women [4-7]. It is believed that a patient may experience different constitutions 
at the same time, and the constitution is dynamically changing with pathological, physiological, and psychological states $[8,9]$.

In 2015, the World Health Organization released the first world report on healthy aging [10]. There is increasing interest in the clustering of risk behaviors in elderly people. However, identifying leading predictors for disease-free status in the middle-age group may provide evidence for action priorities in elderly care. [11, 12]. Middle-aged women experience many physical and mental changes. Previous studies have linked socioeconomic status and lifestyle, behavioral, psychological, and biological factors to healthy aging [13-15]. Body mass index and diet behavior are key factors for disease-free status promotion [11,12,14]. Women living with climacteric symptoms experienced negative physical and psychological consequences that led to changes in their lives, particularly in their relationships [16]. A systematic review shows that menopause is a stage of life experienced in different ways, characterized by personal challenges and changes in personal roles within the family and society. Those women reported hot flushes and night sweats are the strongest symptoms during menopause [17]. Osteoporosis may affect one out of three postmenopausal women. The risk of osteoporosis and fragility fractures can be reduced through healthy lifestyle changes $[18,19]$. Chronic obstructive pulmonary disease and airway obstruction were also related to bone mineral density in postmenopausal women [20]. Those symptoms were consistent with the performance of constitutions in deficiency and stasis $[7,21]$. No study has examined the relationship between health indicators and constitutions.

Both modern medicine and TCM expenses are covered in parallel by the National Health Insurance scheme in Taiwan. More than one-quarter of the people have ever used Chinese medicine in Taiwan, especially women, and this is expected to continue in the future [22-24]. TCM might have a positive impact on the relief of climacteric symptoms and the prevention of osteoporotic fracture [25, 26]. TCM doctors evaluated periodical treatment as an important part of the process of individual treatment based on differentiation of constitution. [27, 28]. No major studies have measured the constitution in middle-age women in Taiwan. Therefore, we have little knowledge of the constitution differences between the deficiency (yin and yang) and stasis. No study has examined the health determinants of constitution. Therefore, the objectives of this study were to describe constitutions in yang deficiency, yin deficiency, and stasis among middle-aged women. In addition, demographic and health factors related to yang deficiency, yin deficiency, and stasis were examined. The results could allow TCM doctors to plan health promotion programs to fit the demands of TCM and personal needs in the follow-up with middle-age women.

\section{Materials and Methods}

This study was based on the analysis of secondary data. All data were provided from the Taiwan Biobank, which collected specimens and relevant information from individuals in recruitment centers across Taiwan. The study was approved by the human subjects committee at MacKay Memorial Hospital in Taipei. All participant names were replaced by coded numbers to ensure anonymity. Written consent was not required since patient identifiers were not included in the data.

2.1. Study Participants. Middle age, as defined in the Oxford Learners Dictionary, is the period between early adulthood and old age, usually considered as the years from about 45 to 60 [29]. The Merriam-Webster Dictionary defines middle age from about 40 to 64 years [30], whereas the prominent psychologist Erik Erikson saw it as starting a little earlier and defines middle adulthood as between 40 and 65 years [31]. Hence, the inclusion criteria in our study were women: (1) age, 40-65 years; (2) study period, 2009 to 2018; (3) completed the survey including diet behavior, female characteristics, health examination, and constitutions. The sample size was estimated using $G^{*}$ Power: Statistical Power Analyses software. Logistic regression was used to compute the required sample size. The $\alpha$ error probability was set to 0.05 ; the power was 0.95 , and the odds ratio was set at 1.3 . The calculated sample size was 988 . Among the 27,366 potential study candidates, a total of 1,000 women aged 40-65 years were selected from 2009 to 2018 using random sampling from the Taiwan Biobank Research Database. Taiwan Biobank aims to build a nationwide research database that conducts large-scale cohort studies and case-control studies on local diseases. About 200,000 volunteers will be called for in the cohort study, while 100,000 patients with the 10 to 15 most common diseases will be invited in the case-control study.

2.2. Measurements. The study variables included demographics (age, marital status, work status, and education), dietary behavior (vegetarian, tea habit, and coffee habit), female characteristics (menstruation, pregnancy, and abortion), health examination (body mass index, waist and hip circumference, bone mineral density, and pulmonary function), and constitutions (yang deficiency, yin deficiency, and stasis).

The Body Constitution Questionnaire (BCQ) was developed to measure psychological and physiological states of deviation in the constitution. The BCQ instrument was developed through a literature review and a Delphi process by 26 experts [32]. The reliability and validity of the scale were confirmed [33-36]. The BCQ included 44 items covering 3 identifications of the constitution: yang deficiency (19 items), yin deficiency (19 items), and stasis (16 items) (see the appendix). Each item was rated on a 5-point frequency or intensity Likert scale, ranging from 1 (never or not at all) to 5 (always or very severe). Assessments of internal validity, internal consistency, test-retest, and criterion-related tests were used to confirm the reliability and validity of the BCQ [30-33].

A yang-deficiency constitution indicates that a person's energy (qi) for maintaining body functions is insufficient, and they may experience symptoms such as fatigue, 
shortness of breath, chills, loose stool, and a large volume of urine. A person was considered to have a yang-deficiency constitution if the sum of the BCQ + scores was $\geq 30.5$ [32,33].

A yin-deficiency constitution means the insufficiency of body fluid, which means a person may present symptoms such as thirst, constipation, oral ulcers, and dry eyes. A total BCQ- score $\geq 29.5$ was considered a yin-deficiency constitution [34]. The stasis constitution features the imbalance and obstruction of qi and blood. People with the stasis may have lesions of pain, numb limbs, sputum, or edema. A patient with a sum of BCQs scores $\geq 26.5$ was considered to have a stasis constitution [35].

The analyzed data included the health examination results obtained for body mass index, waist and hip circumference, bone mineral density, and pulmonary function. Body mass index was calculated as a person's weight in kilograms divided by the square of height in meters. Categories of body mass index included underweight (under $\left.18.5 \mathrm{~kg} / \mathrm{m}^{2}\right)$, normal weight $\left(18.5\right.$ to $\left.25 \mathrm{~kg} / \mathrm{m}^{2}\right)$, overweight (25 to $30 \mathrm{~kg} / \mathrm{m}^{2}$ ), and obese (over $30 \mathrm{~kg} / \mathrm{m}^{2}$ ). The bone mineral density (BMD) $T$ score is used to compare with the healthy young adult (average 30 years old), while the $Z$ score compares an individual's bone density to the average bone density of people her own age and gender. A $T$ (or $Z$ ) score within 1 means the individual's BMD is healthy. People with $T$ (or $Z$ ) score between -1.0 and -2.5 means low bone density or osteopenia. A score lower than -2.5 is a diagnosis of osteoporosis. The results of pulmonary function tests including forced vital capacity (FVC), forced expiratory volume in one second (FEV1), FEV1/FVC ratio, and total lung capacity (TLC) are the indicators to evaluate the obstructive impairment, restrictive impairment, or mixed impairment. Women with normal FVC $(\geq 80 \%)$, FEV1 $(\geq 80 \%$ ), and FEV1/FVC ratio (within $5 \%$ of the predicted ratio), and TLC ( $\geq 80 \%)$ were identified as a normal group of the pulmonary function test in our study.

2.3. Data Analysis. Statistical analyses were performed using the Predictive Analytics Suite workstation running version 18.0 software (PASW, IBM Corp., Somers, New York, USA). Individual variables were examined by percentages, means, and standard deviations. The chi-square test and $t$-test were used to determine the significance of differences in characteristics between the women who had deficiency or stasis constitution or not. We used multiple logistic regression analysis to identify factors associated with deficiencies or stasis constitutions. In all analyses, $P<0.05$ was considered statistically significant.

\section{Results}

3.1. Characteristics of the Participants. The mean age of the study women was 51.98 years $(\mathrm{SD}=6.84)$. More than half of the women were employed $(58.1 \%)$, married $(74.5 \%)$, and had been pregnant (88.9\%). Some women had a habit of drinking tea $(29.1 \%)$ or coffee $(33.9 \%)$. Only $77(7.7 \%)$ women were vegetarian. The number of women with current menstruation in age $<51$ (years), $51 \leq$ age $<56$ (years), and $56 \leq$ age $<65$ (years) groups were $362(86.4 \%), 79(33.3 \%)$, and $4(1.2 \%)$. The percentage of middle-aged women who had the constitution in yang-deficiency, yin-deficiency, and stasis was $29.7 \%(n=297), 21.7 \%(n=217)$, and $17.7 \%$ $(n=177)$, respectively (Table 1$)$. Of the 356 women with one constitution at least, $35.1 \%(n=125)$ women experienced yang-deficiency, yin-deficiency, and stasis constitutions at the same time, $23.8 \%(n=85)$ women had two of three constitutions, and $41.0 \%$ women $(n=146)$ had one of three constitutions.

Among the women, 384 (38.4\%) reported being overweight $(24.4 \%)$ or obese $(14 \%)$. The overall mean waist and hip circumference of the women were $80.88 \mathrm{~cm}(\mathrm{SD}=8.76)$ and $95.26 \mathrm{~cm}(\mathrm{SD}=6.92)$. The $Z$ score for bone mineral density indicated 91 women had osteopenia or osteoporosis. The percentages of normal spirometry, obstructive impairment, restrictive impairment, and mixed impairment were $60 \%(n=600), 22 \%,(n=220) 13.7 \%(n=137)$, and $4.3 \%(n=43)$, respectively (Table 1$)$.

3.2. Factors Related to Yang-Deficiency Constitution. Bivariate analyses showed that age, marital status, education, coffee habit, current menstruation, ever having been pregnant, and abnormal spirometry were significantly associated with the constitution of yang deficiency (Table 1). We included and adjusted for those variables in the multiple logistic regression analyses. Because the results of logistic regression with forward and backward methods showed no significant differences in age, marital status, coffee habit, and ever having been pregnant, we excluded those variables. Considering the possible interaction of age and menstruation status on yang deficiency, the data were divided by age groups to evaluate the menstruation effect on the yangdeficiency constitution. Among the three groups of age, there was no significant difference in the yang-deficiency constitution between women with and without menstruation (Table 2). The final model shows that women with abnormal spirometry had a higher risk for the yang-deficiency constitution $(\mathrm{OR}=1.42,95 \% \mathrm{CI} 1.07-1.88, P=0.02)$. Participants with current menstruation had a greater risk for a yang-deficiency constitution $(\mathrm{OR}=1.45,95 \%$ CI 1.10-1.93, $P<0.01)$. Compared with the people with elementaryschool education, those graduated from high school, university, or graduate school had a greater risk for a yangdeficiency constitution (high school: OR $=2.42,95 \% \mathrm{CI}$ 1.11-5.27, $P=0.03$; college or university: $\mathrm{OR}=2.80,95 \% \mathrm{CI}$ 1.28-6.12, $P=0.01$; graduate school: $\mathrm{OR}=2.75,95 \% \mathrm{CI}$ 1.08-6.97, $P=0.03$ ). Table 3 presents the logistic regression models of the yang-deficiency constitution.

\subsection{Factors Related to Yin-Deficiency Constitution.} Bivariate analyses showed that age, vegetarian diet, coffee habit, current menstruation, bone mineral density $z$ score, and abnormal spirometry were significantly associated with the constitution of yin deficiency (Table 1). We included and adjusted for those variables in the multiple logistic regression analyses. Because the results of logistic regression with 
TABLE 1: Differences in demographic data and health-related variables between women with and without yang deficiency, yin deficiency, and stasis $(n=1000)$.

\begin{tabular}{|c|c|c|c|c|c|c|c|c|}
\hline \multirow{3}{*}{ Variable } & \multirow{2}{*}{\multicolumn{2}{|c|}{ Total }} & \multicolumn{6}{|c|}{ Constitution } \\
\hline & & & \multicolumn{2}{|c|}{$\begin{array}{c}\text { Yang deficiency } \\
(n=297)\end{array}$} & \multicolumn{2}{|c|}{$\begin{array}{l}\text { Yin deficiency } \\
\quad(n=217)\end{array}$} & \multicolumn{2}{|c|}{ Stasis $(n=177)$} \\
\hline & $n$ & $(\%)$ & $N$ & $(\%)$ & $n$ & $(\%)$ & $n$ & $(\%)$ \\
\hline \multicolumn{9}{|l|}{ Age (years) } \\
\hline $40 \leq$ age $<51$ & 419 & $(41.9)$ & 142 & $(47.8)^{*}$ & 102 & $(47.0)^{*}$ & 99 & $(55.9)^{* *}$ \\
\hline $51 \leq$ age $<56$ & 237 & $(23.7)$ & 72 & $(24.2)$ & 59 & $(27.2)$ & 45 & $(25.4)$ \\
\hline $56 \leq$ age $<65$ & 344 & $(34.4)$ & 83 & $(27.9)$ & 56 & $(25.8)$ & 33 & $(18.6)$ \\
\hline \multicolumn{9}{|l|}{ Marital status } \\
\hline Single & 86 & $(8.6)$ & 34 & $(11.5)^{* *}$ & 21 & $(9.8)$ & 22 & $(12.4)^{* *}$ \\
\hline Married & 744 & $(74.5)$ & 206 & $(69.8)$ & 152 & $(70.7)$ & 117 & $(66.1)$ \\
\hline Widowed & 95 & $(9.5)$ & 38 & $(12.9)$ & 28 & $(13.0)$ & 27 & $(15.3)$ \\
\hline Divorced & 73 & $(7.3)$ & 17 & $(5.8)$ & 14 & $(6.5)$ & 11 & $(6.2)$ \\
\hline \multicolumn{9}{|l|}{ Currently working } \\
\hline No & 419 & $(41.9)$ & 114 & $(38.4)$ & 83 & $(38.2)$ & 59 & $(33.3)^{*}$ \\
\hline Yes & 581 & $(58.1)$ & 183 & $(61.6)$ & 134 & $(61.8)$ & 118 & $(66.7)$ \\
\hline \multicolumn{9}{|l|}{ Education } \\
\hline Elementary school & 60 & $(6.0)$ & 8 & $(2.7)^{*}$ & 10 & $(4.6)$ & 4 & $(2.3)$ \\
\hline High school & 464 & $(46.4)$ & 135 & $(45.5)$ & 89 & $(41.0)$ & 79 & $(44.6)$ \\
\hline College or university & 414 & $(41.4)$ & 134 & $(45.1)$ & 105 & $(48.4)$ & 83 & $(46.9)$ \\
\hline Graduate school & 62 & $(6.2)$ & 20 & $(6.7)$ & 13 & $(6.0)$ & 11 & $(6.2)$ \\
\hline \multicolumn{9}{|l|}{ Vegetarian } \\
\hline No & 923 & $(92.3)$ & 271 & $(91.2)$ & 211 & $(97.2)^{* *}$ & 169 & $(95.5)$ \\
\hline Yes & 77 & $(7.7)$ & 26 & $(8.8)$ & 6 & $(2.8)$ & 8 & $(4.5)$ \\
\hline \multicolumn{9}{|l|}{ Tea habit } \\
\hline No & 709 & $(70.9)$ & 204 & $(68.7)$ & 150 & $(69.1)$ & 122 & $(68.9)$ \\
\hline Yes & 291 & $(29.1)$ & 93 & $(31.3)$ & 67 & $(30.9)$ & 55 & $(31.1)$ \\
\hline \multicolumn{9}{|l|}{ Coffee habit } \\
\hline No & 661 & $(66.1)$ & 181 & $(60.9)^{*}$ & 131 & $(60.4)^{*}$ & 101 & $(57.1)^{* *}$ \\
\hline Yes & 339 & $(33.9)$ & 116 & $(39.1)$ & 86 & $(39.6)$ & 76 & $(42.9)$ \\
\hline \multicolumn{9}{|l|}{ Menstruation } \\
\hline No & 555 & $(55.5)$ & 144 & $(48.5)^{* *}$ & 106 & $(48.8)^{*}$ & 70 & $(39.5)^{* *}$ \\
\hline Yes & 445 & $(44.5)$ & 153 & $(51.5)$ & 111 & $(51.2)$ & 107 & $(60.5)$ \\
\hline \multicolumn{9}{|l|}{ Ever pregnant } \\
\hline No & 101 & $(10.1)$ & 38 & $(12.8)^{*}$ & 23 & $(10.6)$ & 21 & (11.9) \\
\hline Yes & 899 & $(89.9)$ & 259 & $(87.2)$ & 194 & $(89.4)$ & 156 & $(88.1)$ \\
\hline \multicolumn{9}{|l|}{ Ever abortion } \\
\hline No & 432 & $(43.2)$ & 124 & $(41.8)$ & 85 & $(39.2)$ & 71 & $(40.1)$ \\
\hline Yes & 568 & $(56.8)$ & 173 & $(58.2)$ & 132 & $(60.8)$ & 106 & $(59.9)$ \\
\hline \multicolumn{9}{|l|}{ Body mass index } \\
\hline Underweight & 21 & $(2.1)$ & 7 & $(2.4)$ & 5 & $(2.3)$ & 4 & $(2.3)$ \\
\hline Normal weight & 595 & $(59.5)$ & 181 & $(60.9)$ & 133 & $(61.3)$ & 98 & $(55.4)$ \\
\hline Overweight & 244 & $(24.4)$ & 72 & $(24.2)$ & 49 & $(22.6)$ & 48 & $(27.1)$ \\
\hline Obese & 140 & $(14)$ & 37 & $(12.5)$ & 30 & $(13.8)$ & 27 & $(15.3)$ \\
\hline \multicolumn{9}{|l|}{ Bone mineral density $T$ score } \\
\hline Normal $(\geq 1.0)$ & 659 & $(65.9)$ & 204 & $(68.7)$ & 154 & $(71.0)$ & 124 & $(70.1)$ \\
\hline Osteopenia $(-2.4$ to -1.1$)$ & 267 & $(26.7)$ & 74 & $(24.9)$ & 52 & $(24.0)$ & 45 & $(25.4)$ \\
\hline Osteoporosis $(\leq-2.5)$ & 74 & $(7.4)$ & 19 & $(6.4)$ & 11 & $(5.1)$ & 8 & $(4.5)$ \\
\hline Bone mineral density $Z$ score & & & & & & & & \\
\hline Normal $(\geq-1.0)$ & 909 & $(90.9)$ & 273 & $(91.9)$ & 203 & $(93.5)^{*}$ & 163 & $(92.1)$ \\
\hline Osteopenia $(-2.4$ to -1.1$)$ & 88 & $(8.8)$ & 22 & $(7.4)$ & 12 & $(5.5)$ & 13 & $(7.3)$ \\
\hline Osteoporosis $(\leq-2.5)$ & 3 & $(0.3)$ & 2 & $(0.7)$ & 2 & $(0.9)$ & 1 & $(0.6)$ \\
\hline Pulmonary function & & & & & & & & \\
\hline Normal spirometry & 600 & $(60)$ & 164 & $(55.2)^{*}$ & 117 & $(53.9)^{*}$ & 93 & $(52.5)^{*}$ \\
\hline Abnormal spirometry ${ }^{a}$ & 400 & $(40)$ & 133 & $(44.8)$ & 100 & $(46.1)$ & 84 & $(47.5)$ \\
\hline
\end{tabular}

Chi-square testing was used to determine the significance of differences in characteristics between the women who had yang-deficiency, yin-deficiency, or stasis constitutions or not; * the significance level was set at $P<0.05 ;{ }^{* *} P<0.01$. ${ }^{a}$ Included obstructive impairment, restrictive impairment, and mixed impairment. 
TABLE 2: Menstruation effect is divided by age groups on the yang-deficiency, yin-deficiency, and stasis constitutions.

\begin{tabular}{|c|c|c|c|c|c|c|c|c|c|}
\hline & \multicolumn{9}{|c|}{ Constitution } \\
\hline & \multicolumn{3}{|c|}{ Yang deficiency } & \multicolumn{3}{|c|}{ Yin deficiency } & \multicolumn{3}{|c|}{ Stasis } \\
\hline & OR & (95\% CI) & $P$ value & OR & $(95 \% \mathrm{CI})$ & $P$ value & OR & $(95 \% \mathrm{CI})$ & $P$ value \\
\hline \multicolumn{10}{|l|}{$40 \leq a g e<51(y r)$} \\
\hline Menstruation $^{a}$ & 1.24 & $(0.68-2.28)$ & 0.49 & 0.89 & $(0.47-1.68)$ & 0.71 & 1.34 & $(0.67-2.71)$ & 0.41 \\
\hline \multicolumn{10}{|l|}{$51 \leq$ age $<56(y r)$} \\
\hline Menstruation $^{a}$ & 1.30 & $(0.73-2.33)$ & 0.37 & 1.39 & $(0.76-2.57)$ & 0.29 & 1.43 & $(0.73-2.80)$ & 0.29 \\
\hline \multicolumn{10}{|l|}{56 age $<65(y r)$} \\
\hline Menstruation $^{a}$ & 1.05 & $(0.11-10.22)$ & 0.97 & 1.73 & $(0.18 .16 .91)$ & 0.64 & 3.21 & $(0.32-31.75)$ & 0.32 \\
\hline
\end{tabular}

Analysis by logistic regression. $P<0.05$ being statistically significant. OR: odds ratio. CI: confidence interval. Reference group: ${ }^{a}$ menopause.

TABLE 3: Logistic regression model results in yang-deficiency, yin-deficiency, and stasis constitutions.

\begin{tabular}{|c|c|c|c|c|c|c|}
\hline & $B$ & S.E. & Wald & $P$ value & OR & $95 \% \mathrm{CI}$ \\
\hline \multicolumn{7}{|l|}{ Yang deficiency } \\
\hline Abnormal spirometry ${ }^{a}$ & 0.35 & 0.14 & 5.97 & 0.02 & 1.42 & $1.07-1.88$ \\
\hline Menstruation $^{b}$ & 0.37 & 0.14 & 6.85 & $<0.01$ & 1.45 & $1.10-1.93$ \\
\hline High school $^{c}$ & 0.89 & 0.40 & 4.98 & 0.03 & 2.42 & $1.11-5.27$ \\
\hline College or university ${ }^{c}$ & 1.03 & 0.40 & 6.67 & 0.01 & 2.80 & $1.28-6.12$ \\
\hline Graduate school $^{c}$ & 1.01 & 0.48 & 4.53 & 0.03 & 2.75 & $1.08-6.97$ \\
\hline \multicolumn{7}{|l|}{ Yin deficiency } \\
\hline $40 \leq$ age $<51(\mathrm{yr})^{d}$ & 0.59 & 0.19 & 9.85 & $<0.01$ & 1.81 & $1.25-2.62$ \\
\hline $51 \leq$ age $<56(\mathrm{yr})^{d}$ & 0.59 & 0.21 & 7.65 & $<0.01$ & 1.80 & $1.19-2.72$ \\
\hline Abnormal spirometry ${ }^{a}$ & 0.39 & 0.16 & 6.02 & 0.01 & 1.48 & $1.08-2.01$ \\
\hline Vegetarian $^{e}$ & -1.29 & 0.43 & 8.78 & $<0.01$ & 0.28 & $0.12-0.65$ \\
\hline \multicolumn{7}{|l|}{ Stasis } \\
\hline $40 \leq$ age $<51(\mathrm{yr})^{d}$ & 1.13 & 0.22 & 26.18 & $<0.01$ & 3.08 & $2.00-4.75$ \\
\hline $51 \leq$ age $<56(\mathrm{yr})^{d}$ & 0.83 & 0.25 & 11.04 & $<0.01$ & 2.29 & $1.41-3.74$ \\
\hline Abnormal spirometry ${ }^{a}$ & 0.49 & 0.17 & 8.08 & $<0.01$ & 1.63 & $1.16-2.28$ \\
\hline Coffee habit ${ }^{f}$ & 0.40 & 0.17 & 5.26 & 0.02 & 1.49 & $1.06-2.08$ \\
\hline
\end{tabular}

Multivariate logistic regression analysis was performed. $P<0.05$ being statistically significant. OR: odds ratio. CI: confidence interval. Reference group: ${ }^{a}$ normal spirometry; ${ }^{b}$ menopause; ${ }^{c}$ elementary school; ${ }^{d} 56 \leq$ age $<65$ (yr.); ${ }^{e}$ not vegetarian; ${ }^{f}$ without coffee habit.

forward and backward methods showed no significant differences for coffee habit, current menstruation, and bone mineral density $z$ score, we excluded those variables. Considering the possible interaction of age and menstruation status on yin deficiency, the data were divided by age groups to evaluate the menstruation effect on the yindeficiency constitution. Among the three groups of age, there was no significant difference in the yin-deficiency constitution between women with and without menstruation (Table 2). The final model shows that women younger than 56 years had a higher risk of yin-deficiency constitution $(40 \leq \mathrm{Age}<51$ : $\mathrm{OR}=1.81,95 \%$ CI $1.25-2.62$, $P<0.01 ; \quad 51 \leq$ Age $<56: \quad$ OR $=1.80$, 95\% CI 1.19-2.72, $P<0.01)$. Participants with abnormal spirometry also had a greater risk for the yin-deficiency constitution $(\mathrm{OR}=1.48,95 \%$ CI $1.08-2.01, P=0.01)$. Vegetarians in our study had a lower risk for the yin-deficiency constitution $(\mathrm{OR}=0.28,95 \% \mathrm{CI} 0.12-0.65, P<0.01)$. Table 3 presents the logistic regression models of the yin-deficiency constitution.

3.4. Factors Related to Stasis Constitution. Bivariate analyses showed that age, marital status, currently working, coffee habit, current menstruation, and abnormal spirometry were significantly associated with the stasis constitution (Table 1). We included and adjusted for those variables in the multiple logistic regression analyses. Because the results of logistic regression with forward and backward methods showed no significant differences in marital status, current menstruation, and currently working, we excluded those variables. Considering the possible interaction of age and menstruation status on stasis, the data were divided by age groups to evaluate the menstruation effect on the stasis constitution. Among the three groups of age, there was no significant difference in stasis constitution between women with and without menstruation (Table 2). The final model shows that women younger than 56 years had a higher risk for a stasis constitution $(40 \leq \mathrm{Age}<51$ : $\mathrm{OR}=3.08,95 \% \mathrm{CI}$ $2.00-4.75, \quad P<0.01 ; \quad 51 \leq \mathrm{Age}<56: \quad \mathrm{OR}=2.29, \quad 95 \% \mathrm{CI}$ $1.41-3.74, P<0.01)$. Participants with abnormal spirometry also had a greater risk for stasis constitution $(\mathrm{OR}=1.63,95 \% \mathrm{CI} 1.16-2.28, P<0.01)$. There was a greater risk for a stasis constitution in women with a coffee habit $(\mathrm{OR}=1.49,95 \%$ CI $1.06-2.08, P=0.02)$. Table 3 presents the logistic regression models of the stasis constitution. 


\section{Discussion}

We found that the yang-deficiency, yin-deficiency, and stasis constitutions were common in the middle-aged women, especially the yang-deficiency constitution. Of all middleaged women, one in five suffered at least two constitutions at the same time. The result is consistent with TCM theory [1-3]. The simultaneous presence of two constitutions in an individual is like the concept of comorbidity in modern medicine. A constitution may include several diseases, whereas a disease can be expressed in different syndromes. As a disease advances, constitutions may transform into different zhengs $[3,37]$. TCM doctors should be aware of the cooccurrence of deficiency and stasis among middle-aged women and focus their treatment on restoring balance to support their health.

We found that middle-aged women with current menstruation had deteriorating yang-deficiency constitution, whereas women younger than 56 years experienced worse yin-deficiency and stasis constitutions. This result is consistent with a previous study, which indicated the yindeficiency, yang-deficiency, and stasis constitutions were significantly worse in perimenopausal and postmenopausal than premenopausal women with climacteric syndrome [7]. More than $21 \%$ of the climacteric women had ever visited TCM doctors [38]. Eclipta prostrata with Ligustrum lucidum and dan-zhi-xiao-yao-san were the most commonly used combination of Chinese herbal products by TCM doctors in Taiwan [39]. Nonetheless, more study is needed to examine the effectiveness of TCM treatment, such as scrapping, acupuncture, or herbal therapies, based on constitution classification among women with climacteric syndrome [39-41].

Drinking four or more cups of coffee per day is associated with a higher hip fracture risk, whereas moderate intake may alleviate the risk in postmenopausal women [42]. In our study, a coffee habit was also associated with an increased stasis constitution among the study participants. The stasis constitution manifests itself as fatigue, headache with pressure, or pain. Those symptoms could be associated with the motivation for drinking coffee. TCM doctors are expected to assess the causality between coffee drinking and stasis constitution. Suggesting a moderate intake of coffee might help reduce the stasis constitution and avoid worsening osteoporosis. In the study, osteoporosis was not significantly related to deficiency and stasis constitutions, which could be due to the small number of patients with osteoporosis. Besides, patients with osteoporosis tended to take more supplements. Future study is needed to examine the effectiveness of TCM treatment on decreasing other constitutions among patients with osteoporosis.

We found that women with abnormal spirometry had deteriorating the constitutions in yin-deficiency, yang-deficiency, and stasis. Some evidence indicates that TCM treatment could help alleviate pulmonary disease [43-45]. For example, the active agents of single herbs and TCM formulas, particularly the flavonoids, glycosides, and alkaloids, reduced pulmonary fibrosis [43]. Based on the clinical effectiveness of TCM, potential target discovery for asthma treatment is also a feasible strategy [44]. More research is needed to clarify the unconfirmed chemical composition and regulatory mechanisms, conduct standard clinical trials, and evaluate the possible side effects of these TCM herbs and formulations. The use of constitutions as an outcome indicator in the treatment of pulmonary disease is worth future studies [45].

The yin-deficiency constitution is significantly correlated with hypertension and diabetes mellitus [46]. Our finding indicates women with a vegetarian diet had a lower risk for the yin-deficiency constitution. It implies reducing the chance of a yin-deficiency constitution through a vegetarian diet may lower the risk of cardiovascular disease or diabetes mellitus. Some cross-sectional studies also indicate the vegetarian diet is associated with a lower fasting insulin level, higher insulin sensitivity, higher serum concentrations of polyunsaturated, higher monosaturated fatty acids, lower saturated fatty acids, lower long chain omega-3, lower trans fatty acids, and better renal function [47-49]. The vegetarian diet is also negatively associated with diabetes and impaired fasting glucose in premenopausal women and in menopausal women [50]. The experimental and longitudinal studies are suggested to explore the causal relationship between the yin-deficiency constitution and cardiovascular disease or diabetes mellitus among vegetarians, especially for indicators from blood tests. In addition, our result shows women with a high-school education or above have a higher risk of yang-deficiency constitution compared to those with elementary-school education. A survey indicates a significantly positive correlation existed between the yang-deficiency constitution and favor for hot food, and a negative correlation existed between regular exercise yang-deficiency constitution [51]. Collecting more data, such as different habits of diet and exercise, in middle-aged women, may be helpful to clarify the relationship between education level and yang-deficiency constitution.

Although we recruited participants from the Taiwan Biobank, our study did not include all possible participants because of the limited budget. Randomized sampling might have reduced potential selection bias. Women who did not participate in the Taiwan Biobank Survey might differ from those who took part in the survey and became a member of Taiwan Biobank. Hence, our results may not be generalizable outside of the Taiwan Biobank participants. Additionally, our findings are based on the analysis of secondary data. The secondary data analysis with a cross-sectional survey in our study could not provide the causality effect. Causality between variables and their associated constitutions becomes imperative for future prospective studies. Though BCQ revealed acceptable reliability and validity in previous studies [32-36], whether measuring the change of constitutions from longitudinal studies was consistent with the result in our study merits further study. With a stratified analysis of age, our findings show that there is no significant relationship between menstruation and deficiency/stasis constitution. It may be attributed to the lack of interaction between age and menstruation on deficiency/stasis constitution or small sample size in our study. The measurement with cutoff points of three constitutions in BCQ was applied 
TABLE 4: Items and identifications of yang-deficiency, yin-deficiency, and stasis constitutions in body constitution questionnaire (BCQ).

\begin{tabular}{lccc}
\hline Constitution & Total items & Item number & Cutoff score \\
\hline Yang deficiency & 19 & $3,5,8,9,15,16,17,22,23,24,28,31,33,36,37,41,42,43,44$. & 30.5 \\
Yin deficiency & 19 & $2,4,8,10,11,16,18,20,23,26,29,30,31,32,35,37,38,39,40$. & 29.5 \\
Stasis & 16 & $1,4,5,6,7,12,13,14,16,17,19,20,21,25,27,34$. & 26.5 \\
\hline
\end{tabular}

in previous research [52-54] and our study. The method may be underestimating the change of constitutions. We measured only the deficiencies of yin and yang and stasis constitutions in middle-age women, and the study of more constitutions to measure women's overall health status are suggested for future studies.

\section{Conclusion}

Yin deficiency, yang deficiency, and stasis were common in middle-age women in Taiwan. Women with abnormal spirometry have had a higher risk for the constitutions in yin and yang deficiencies and stasis. Participants with current menstruation had a greater risk for a yang-deficiency constitution, while those younger than 56 years had a higher risk of yin-deficiency and stasis constitution. TCM doctors should focus their treatment on simultaneously balancing both deficiency and stasis constitutions. Future study is needed to examine the changes in deficiency and stasis constitutions among patients with climacteric symptoms or respiratory-related diseases or both.

\section{Appendix}

Identifications of yang-deficiency, yin-deficiency, and stasis constitutions in body constitution questionnaire (BCQ): see Table 4.

References from:

(1) L. L. Chen, J. S. Lin, J. D. Lin, et al., "BCQ+: a body constitution questionnaire to assess Yang-Xu. Part II: evaluation of reliability and validity," Forsch Komplementmed, vol. 16, no. 1, pp. 20-27, 2009. doi: 10.1159/000197770.

(2) J. S. Lin, L. L. Chen, J. D. Lin, et al., "BCQ-: a body constitution questionnaire to assess Yin-Xu Part II: evaluation of reliability and validity," Forschende Komplementärmedizin/Research in Complementary Medicine, vol. 19, no. 6, pp. 285-292, 2012. doi: 10.1159/000346060.

(3) J. D. Lin, J. S. Lin, L. L. Chen, et al., "BCQs: a body constitution questionnaire to assess stasis in traditional Chinese medicine," European Journal of Integrative Medicine, vol. 4, no. 4, pp. e379e391, 2012. doi: 10.1016/j.eujim.2012.05.001.

(4) C. H. Lee, T. C. Li, C. I. Tsai, et al., "Association between albuminuria and different body constitution in type 2 diabetes patients: Taichung diabetic body constitution study," Evidence-Based Complementary and Alternative Medicine, vol. 2015, Article ID 603048, 8 pages, 2015. doi: 10.1155/2015/603048.
(5) C. H. Lee, T. C. Li, C. I. Tsai, et al., "Yang deficiency body constitution acts as a predictor of diabetic retinopathy in patients with type 2 diabetes: Taichung diabetic body constitution study," EvidenceBased Complementary and Alternative Medicine, vol. 2015, Article ID 940898, 8 pages, 2015. doi: 10.1155/ 2015/940898.

\section{Data Availability}

The datasets used and/or analyzed during the current study are available from the corresponding author on reasonable request.

\section{Ethical Approval}

The study was approved by the human subjects committee at one hospital in Taipei (19MMHIS164e).

\section{Conflicts of Interest}

The authors declare no conflicts of interest.

\section{Authors' Contributions}

PHC and SMH designed the study. JCYL performed the statistical analysis. An initial draft of the manuscript was written by $\mathrm{SMH}$. All authors were involved in writing the manuscript. Each author has read and approved the final manuscript.

\section{Acknowledgments}

The authors thank Taiwan Biobank who provided anonymous data. This study was supported by the Office of Research and Development, Mackay Medical College, Taiwan (grant no. 1081B04).

\section{References}

[1] W. Yu, M. Ma, X. Chen et al., "Traditional Chinese medicine and constitutional medicine in China, Japan and Korea: a comparative study," The American Journal of Chinese Medicine, vol. 45, no. 1, pp. 1-12, 2017.

[2] A. S. Min and A. J. Lopes, "Chinese medicine pattern differentiation and its implications for clinical practice," Chinese Journal of Integrative Medicine, vol. 17, no. 11, pp. 818-823, 2011.

[3] Q. Wang, "Individualized medicine, health medicine, and constitutional theory in Chinese medicine," Frontiers of Medicine, vol. 6, no. 1, pp. 1-7, 2012.

[4] World Health Organization, "Regional office for the western pacific. WHO international standard terminologies on traditional medicine in the western pacific region," WHO 
Regional Office for the Western Pacific, Manila, Philippines, 2007, https://apps.who.int/iris/handle/10665/206952.

[5] H. M. Langevin, G. J. Badger, B. K. Povolny et al., "Yin scores and yang scores: a new method for quantitative diagnostic evaluation in traditional Chinese medicine research," The Journal of Alternative and Complementary Medicine, vol. 10, no. 2, pp. 389-395, 2004.

[6] C. L. Davis, S. F. Kuo, Q. Y. Chang et al., "Pattern identification of qi deficiency and blood stasis in cancer patients," Mid-Taiwan Journal of Medicine, vol. 2, no. 2, pp. 109-116, 2007.

[7] H.-C. Wu, K.-H. Chen, and J.-S. Hwang, "Association of menopausal symptoms with different constitutions in climacteric women," Complementary Medicine Research, vol. 25, no. 6, pp. 398-405, 2018.

[8] C. A. Berle, D. Cobbin, N. Smith, and C. Zaslawski, "A novel approach to evaluate Traditional Chinese Medicine treatment outcomes using pattern identification," The Journal of Alternative and Complementary Medicine, vol. 16, no. 4, pp. 357-367, 2010.

[9] H. Z. Zaslawski, Q. C. Fan, G. E. Bai et al., "Syndrome differentiation of Chinese medicine in mars 500 long-term closed environment," Chinese Journal of Integrative Medicine, vol. 26, no. 6, pp. 428-433, 2020.

[10] J. R. Beard, A. Officer, I. A. de Carvalho et al., "The World report on ageing and health: a policy framework for healthy ageing," The Lancet, vol. 387, no. 10033, pp. 2145-2154, 2016.

[11] T. D. Sadana, A. M. Prina, J. Perales, B. C. M. Stephan, and C. Brayne, "Operational definitions of successful aging: a systematic review," International Psychogeriatrics, vol. 26, no. 3, pp. 373-381, 2014.

[12] X. Stephan, W. Wang, S. Keel et al., "Leading determinants for disease-free status in community-dwelling middle-aged men and women: a 9-year follow-up cohort study," Frontiers in Public Health, vol. 7, p. 320, 2019.

[13] L. Lafortune, S. Martin, S. Kelly et al., "Behavioural risk factors in mid-life associated with successful ageing, disability, dementia and frailty in later life: a rapid systematic review," PLoS One, vol. 11, no. 2, p. e0144405, 2016.

[14] M. A. Muga, P. O. Owili, C. Y. Hsu et al., “Association between dietary patterns and cardiovascular risk factors among middle-aged and elderly adults in Taiwan: a population-based study from 2003 to 2012," PLoS One, vol. 11, no. 7, p. e0157745, 2016.

[15] C. Samieri, Q. Sun, M. K. Townsend et al., "The association between dietary patterns at midlife and health in aging," Annals of Internal Medicine, vol. 159, no. 9, pp. 584-591, 2013.

[16] P. Giannouli, I. Zervas, E. Armeni et al., "Determinants of quality of life in Greek middle-age women: a population survey," Maturitas, vol. 71, no. 2, pp. 154-161, 2012.

[17] L. Koundi, J. Rodolpho, B. Gonçalves, and B. Quirino, "Women's experience of menopause: a systematic review of qualitative evidence," JBI Database of Systematic Reviews and Implementation Reports, vol. 13, no. 8, pp. 250-337, 2015.

[18] R. Quirino, H. Bischoff-Ferrari, B. Dawson-Hughes, and C. Weaver, "Nutrition and bone health in women after the menopause," Women's Health, vol. 10, no. 6, pp. 599-608, 2014.

[19] S. Weaver and V. Chan, "Women living with osteoporosis: a meta-synthesis," The Gerontologist, 2019, In press.

[20] R. Xu, Y. Zhang, X. C. Chen et al., "Association of bone mineral density with chronic obstructive pulmonary disease in postmenopausal women," Revista de Investigacion Clinica, vol. 71, no. 3, pp. 204-210, 2019.
[21] W. Q. Chen, "TCM clinical treatment women with menopause," The Journal of Traditional Medicine, vol. 26, no. 1, pp. 33-46, 2015, in Chinese.

[22] M. J. Lin, H. W. Chen, P. H. Liu et al., "The prescription patterns of traditional Chinese medicine for women with polycystic ovary syndrome in Taiwan: a nationwide population-based study," Medicine, vol. 98, no. 24, p. e15890, 2019.

[23] C. C. Shih, L. H. Huang, C. C. Yeh et al., "The prevalence, characteristics, and factors associated with purchasing Chinese herbal medicine among adults in Taiwan," BMC Complementary and Alternative Medicine, vol. 17, no. 1, p. 169, 2017.

[24] Y. H. Yeh, Y. J. Chou, N. Huang, C. Pu, and P. Chou, “The trends of utilization in traditional Chinese medicine in Taiwan from 2000 to 2010: a population-based study," Medicine, vol. 95, no. 27, p. e4115, 2016.

[25] S. Eisenhardt and J. Fleckenstein, "Traditional Chinese medicine valuably augments therapeutic options in the treatment of climacteric syndrome," Archives of Gynecology and Obstetrics, vol. 294, no. 1, pp. 193-200, 2016.

[26] Y. C. Wang, J. H. Chiang, H. C. Hsu, and C-H. Tsai, "Decreased fracture incidence with traditional Chinese medicine therapy in patients with osteoporosis: a nationwide population-based cohort study," BMC Complementary and Alternative Medicine, vol. 19, no. 1, p. 42, 2019.

[27] M. Shen, Y. Cui, M. Hu, and X. Linyong, "Quantifying traditional Chinese medicine patterns using modern test theory: an example of functional constipation," BMC Complementary and Alternative Medicine, vol. 17, no. 1, p. 44, 2017.

[28] Y. H. Zhang, J. Lv, W. Gao et al., "Practitioners' perspectives on evaluating treatment outcomes in traditional Chinese medicine," BMC Complementary and Alternative Medicine, vol. 17, no. 1, p. 269, 2017.

[29] Oxford English Dictionary, "The definition of middle age," https://www.oxfordlearnersdictionaries.com/definition/ english/middle-age.

[30] Merriam-Webster, "The definition of middle age," 2020, https://www.merriam-webster.com/dictionary/middle\% 20age.

[31] Wikipedia, "The definition of middle age," https://en. wikipedia.org/wiki/Middle_age.

[32] Y.-C. Su, L.-L. Chen, J.-D. Lin, J.-S. Lin, Y.-c. Huang, and J.-S. Lai, "BCQ+: a body constitution questionnaire to assess Yang-Xu," Complementary Medicine Research, vol. 15, no. 6, pp. 327-334, 2008.

[33] L. L. Chen, J. S. Lin, J. D. Lin et al., "BCQ+: a body constitution questionnaire to assess Yang-Xu. Part II: evaluation of reliability and validity," Forschende Komplementarmedizin, vol. 16, no. 1, pp. 20-27, 2016.

[34] J.-S. Lin, L.-L. Chen, J.-D. Lin et al., "BCQ-: a body constitution questionnaire to assess Yin-Xu," Forschende Komplementärmedizin/Research in Complementary Medicine, vol. 19, no. 6, pp. 285-292, 2012.

[35] J.-D. Lin, J.-S. Lin, L.-L. Chen, C.-H. Chang, Y.-C. Huang, and Y.-C. Su, "BCQs: a body constitution questionnaire to assess stasis in traditional Chinese medicine," European Journal of Integrative Medicine, vol. 4, no. 4, pp. e379-e391, 2012.

[36] W. Wong, C. L. K. Lam, Y.-C. Su et al., "Measuring body constitution: validation of the body constitution questionnaire (BCQ) in Hong Kong," Complementary Therapies in Medicine, vol. 22, no. 4, pp. 670-682, 2014. 
[37] M. F. Mei, "A systematic analysis of the theory and practice of syndrome differentiation," Chinese Journal of Integrative Medicine, vol. 17, no. 11, pp. 803-810, 2011.

[38] Y.-H. Yang, P.-C. Chen, J.-D. Wang, C.-H. Lee, and J.-N. Lai, "Prescription pattern of traditional Chinese medicine for climacteric women in Taiwan," Climacteric, vol. 12, no. 6, pp. 541-547, 2009.

[39] H.-Y. Chen, Y.-H. Lin, J.-C. Wu et al., "Prescription patterns of Chinese herbal products for menopausal syndrome: analysis of a nationwide prescription database," Journal of Ethnopharmacology, vol. 137, no. 3, pp. 1261-1266, 2011.

[40] F. Meng, P. Duan, Q. Hu et al., "Scrapping therapy combined with Qingxin Zishen Decoction for perimenopausal syndrome with pattern of fire excess from yin deficiency," Zhongguo Zhen Jiu (Chinese Acupuncture \& Moxibustion), vol. 36, no. 8, pp. 821-826, 2016, in Chinese.

[41] N. E. Avis, C. Legault, R. R. Coeytaux et al., "A randomized, controlled pilot study of acupuncture treatment for menopausal hot flashes," Menopause, vol. 15, no. 6, pp. 1070-1078, 2008.

[42] Z. Dai, A. Jin, A. Z. Soh, L.-W. Ang, J.-M. Yuan, and W.-P. Koh, "Coffee and tea drinking in relation to risk of hip fracture in the Singapore Chinese health study," Bone, vol. 112, pp. 51-57, 2018.

[43] L.-C. Li and L.-D. Kan, "Traditional Chinese medicine for pulmonary fibrosis therapy: progress and future prospects," Journal of Ethnopharmacology, vol. 198, pp. 45-63, 2017.

[44] Y. Wang, Y. J. Chen, C. Xiang et al., "Discovery of potential asthma targets based on the clinical efficacy of traditional Chinese medicine formulas," Journal of Ethnopharmacology, vol. 252, p. 112635, 2020.

[45] X.-q. Yu, S.-g. Yang, Y. Xie, and J.-s. Li, “Traditional Chinese medicine in the treatment of idiopathic pulmonary fibrosis based on syndrome differentiation: study protocol of an exploratory trial," Journal of Integrative Medicine, vol. 18, no. 2, pp. 163-168, 2020.

[46] Y. Zhu, H. Shi, Q. Wang et al., "Association between nine types of TCM constitution and five chronic diseases: a correspondence analysis based on a sample of 2,660 participants," Evidence-based Complementary and Alternative Medicine, vol. 2017, Article ID 9439682, 7 pages, 2017.

[47] X. Cui, B. Wang, Y. Wu et al., "Vegetarians have a lower fasting insulin level and higher insulin sensitivity than matched omnivores: a cross-sectional study," Nutrition, Metabolism and Cardiovascular Diseases, vol. 29, no. 5, pp. 467-473, 2019.

[48] H. Y. Lee, J. Woo, Z. Y. Chen, S. F. Leung, and X. H Peng, "Serum fatty acid, lipid profile and dietary intake of Hong Kong Chinese omnivores and vegetarians," European Journal of Clinical Nutrition, vol. 54, no. 10, pp. 768-773, 2000.

[49] K. Xu, X. Cui, B. Wang et al., "Healthy adult vegetarians have better renal function than matched omnivores: a cross-sectional study in China," BMC Nephrology, vol. 21, no. 1, p. 268, 2020.

[50] T. H. Chiu, H. Y. Huang, Y. F. Chiu et al., "Taiwanese vegetarians and omnivores: dietary composition, prevalence of diabetes and IFG," PLoS One, vol. 9, no. 2, Article ID e88547, 2014.

[51] M. Shi, Z. L. Liu, M. Y. Xu et al., "Relationship between life habits and constitution types of Chinese medicine in the senile living at home in Beijing downtown," Chinese Journal of Integrated Traditional and Western Medicine, vol. 36, no. 5, pp. 564-569, 2016.
[52] C. H. Lee, T. C. Li, C. I. Tsai et al., "Association between albuminuria and different body constitution in type 2 diabetes patients: Taichung diabetic body constitution study," Evidence-based Complementary and Alternative Medicine, vol. 2015, Article ID 603048, 8 pages, 2015.

[53] C. H. Lee, T. C. Li, C. I. Tsai et al., "Yang deficiency body constitution acts as a predictor of diabetic retinopathy in patients with type 2 diabetes: Taichung diabetic body constitution study," Evidence-based Complementary and Alternative Medicine, vol. 2015, Article ID 940898, 8 pages, 2015.

[54] H. J. Chen, Y. J. Lin, P. C. Wu et al., "Study on Yang-Xu using body constitution questionnaire and blood variables in healthy volunteers," Evidence-based Complementary and Alternative Medicine, vol. 2016, Article ID 9437382, 7 pages, 2016. 